\title{
MS4a6D Exacerbates Immunological Pathology in Experimental Viral Fulminant Hepatitis
}

\author{
Jianzhao Deng ${ }^{1}$, Xuan Yang ${ }^{1}$, Bei Zhang ${ }^{1}$
}

\author{
${ }^{1}$ Department of Immunology, Qingdao University, 308 NingXia Road, Qingdao 266071, China
}

\begin{abstract}
The MS4A (membrane-spanning 4-domain family, subfamily A) family of proteins contains some members, including MS4A1, MS4A2 and MS4A3 and so on. Many MS4A family members are expressed on the cell surface of specific leukocyte subsets. And they have key roles in regulating cell activation, growth and development. However, the biological roles of many MS4A proteins is not particularly clear, such as MS4a6D. Here, we found that MS4a6D are expressed on the cell surface of Macrophages and DCs. In MHV3-infected MS4a6 $\mathrm{D}^{-/-}$mice, macrophages decreased, in which the pro-inflammatory subsets decreased than their wild-type (WT) littermates. And the deletion of MS4a6D is associated with less severe damage and less viral replication in the liver. In conclusion, our results suggest that MS4a6D signaling can aggravate the immunopathological damage induced by MHV-3 infection in a mouse FH model. Our results suggest that inhibition of MS4a6D signaling pathways by an immunotherapeutic approach might be a useful treatment for FH.
\end{abstract}

Keywords: MHV3, MS4a6D, FH

\begin{abstract}
Introduction
Viral hepatitis B and C infections are serious health problems affecting over 350 million and 170 million people worldwide. Viral fulminant hepatitis $(\mathrm{FH})$ is a clinical syndrome characterized by massive necrosis of hepatocytes along with hepatic encephalopathy during the infections ${ }^{[1]}$. Although liver transplantation and the development of antiviral drugs has emerged as the effective treatment for fulminant virus hepatitis $(\mathrm{FH})$, the mortality rates associated with $\mathrm{FH}$ are still very high ${ }^{[2]}$. Because a poor understanding of the immune mechanisms underlying viral FH has largely stalled the identification of effective clinical interventions. It has been suggested that the balance between protection and liver damage regulated by the host's immune response will be the decisive factor in the outcome of hepatitis during acute infection. Thus, it appears crucial to dentify the factors of the host's immune response to limit hepatocyte injury.
\end{abstract}

Murine hepatitis virus strain-3 (MHV-3) is a single-stranded, positive-sense RNA virus that belongs to the Coronaviridae family ${ }^{[3]}$. MHV-3-induced FH exhibits a syndrome that is very similar to the clinical situations of patients with viral FH. A mouse FH model, based upon infection with the murine hepatitis virus strain-3 (MHV-3), has provided insights into mechanisms underlying the disease pathogenesis and led to some novel treatment strategies ${ }^{[4]}$. The virus produces strain-dependent disease profiles that depend on the infection route, age, genetic background, and immune status of the host. For example, Balb/c, C57BL/6 and DBA/2 mice develop acute fulminant hepatitis, while mice of the A strain exhibit no evidence of hepatitis ${ }^{[5,6]}$. In contrast to the resistant A strain mice, FH induced by MHV-3 in susceptible mice is characterized by the presence of hepatocellular necrosis and higher viral replication $^{[3,7]}$.

Cells require a variety of surface molecules to work together to deliver appropriate signals in response to environmental stimuli. The MS4A family was first identified based on the observation that the pan B-cell marker, MS4A1 (CD20) and the high-affinity immunoglobulin (Ig)E receptor $\beta$ subunit (MS4A2 or FceRI $\beta$ ) shared a high amino-acid sequence identity ${ }^{18}$, ${ }_{91}$. Subsequently, MS4A3 (HTm4) was cloned ${ }^{[10,}{ }^{11]}$. The results promoted cloning and identification of other MS4A family members in humans and mouse $^{[12,13]}$. The proteins contained in the MS4A family were predicted to span the membrane four times and have similarities in the polypeptide sequences ${ }^{[14-17]}$. There have been many studies on the expression and function of MS4A1, MS4A2, MS4A3, MS4A4B, MS4A8, and MS4A12 ${ }^{[18]}$. And they have been well characterized as having key roles in regulating cell activation, growth and development ${ }^{[19-22]}$. However, beyond these members there are a large number of related molecules where our understanding of their biological roles is at a relatively nascent stage. And little research has been

This article is published under the terms of the Creative Commons Attribution License 4.0 Author(s) retain the copyright of this article. Publication rights with Alkhaer Publications.

Published at: http://www.ijsciences.com/pub/issue/2019-02/

DOI: 10.18483/ijSci.1916; Online ISSN: 2305-3925; Print ISSN: 2410-4477 
done on the MS4a6D gene. We found that there are only two related literatures are available ${ }^{[23,24]}$.

Here, we found that MS4a6D is a membrane protein molecule expressed on the surface of antigen presenting cells such as macrophages and DCs. Given that macrophages play an important role in fighting acute inflammation, and infection with hepatitis $\mathrm{A}, \mathrm{B}$, and $\mathrm{C}(\mathrm{HAV}, \mathrm{HBV}$, and $\mathrm{HCV}$, respectively) remains a substantial health problem all over the world. New HAV and HBV infections have been prevented by the adoption of universal infant vaccination in many countries, but acute infections continue to occur and cause substantial morbidity and mortality ${ }^{[25-27]}$. So we wanted to test how the MS4a6D gene works in mice with acute hepatitis. we suspected that the deletion of MS4a6D would disturb the dynamic balance of macrophages and further affect the ability of mice to resist the viru. This may provide new ideas and methods for research and treatment of hepatitis.

\section{Materials and Methods \\ Mice \\ The wild type (WT) mice were on C57BL/6 background and were purchased from the Jackson Laboratory (Bar Harbor, Maine, USA). Mice were maintained in the animal facility, fed with standard laboratory chow diet and water, and housed in the animal colony at the animal center of Qingdao University. Mice of approximately 12 weeks of age were used for these experiments.}

\section{Virus and infection}

MHV-3 viruses were amplified in murine 17CL1 cells to a concentration of $1 \times 10^{7}$ plaque forming unit $(\mathrm{PFU}) / \mathrm{ml}$. The supernatants containing the virus were stored at $-80^{\circ} \mathrm{C}$ until use. Wild-type (WT) C57BL/6 or MS4a6D ${ }^{-1-}$ mice were injected with $100 \mathrm{PFU}$ of MHV-3 via intraperitoneal injection. Mice were euthanized at $24 \mathrm{~h}$ and $48 \mathrm{~h}$ p.i., and tissues were isolated for hematoxylin and eosin (H\&E) staining to detect damage. In order to monitor the mortality, WT and MS4a6D ${ }^{-/}$mice were injected with $100 \mathrm{PFU}$ of MHV-3 via i.p, then we recorded the number of deaths per day.

\section{Immunohistochemical and immunofluorescence staining}

After 48 and 72 hours of mice injected in the highly virulent mouse hepatitis virus type 3(MHV3), liver tissues are collected after euthanizing the animals and fixed in $4 \%$ paraformaldehyde for more than $24 \mathrm{~h}$. Specimens of liver were dehydrated in a series of graded alcohols. The specimens were embedded in paraffin blocks after a xylene treatment. Paraffin-embedded liver tissue blocks were cut into $3 \mu \mathrm{m}$ slices and mounted onto poly-lysinecharged glass slides. Endogenous peroxidase activity was blocked by exposure to $3.0 \% \mathrm{H}_{2} \mathrm{O}_{2}$ for $15 \mathrm{~min}$. Antigen retrieval was performed in a citrate buffer
$\left(\mathrm{pH} 6.0\right.$ ) at $120^{\circ} \mathrm{C}$ for $10 \mathrm{~min}$. Sections were then incubated at $4^{\circ} \mathrm{C}$ overnight with $\mathrm{B} 220, \mathrm{CD} 4, \mathrm{CD} 8$, CD11c (1:50, rabbit IgG, Santa Cruz), F4/80, Gr-1, NK1.1, ecamp or MS4a6D. Immunoreactivity was detected by using a fluorescein isothiocyanate (FITC)-conjugated (1:100, Zymed, San Francisco, CA, USA) or Cy3-conjugated secondary antibodies (1:200; Jackson ImmunoResearch, West Grove, PA, USA). Results were analyzed by fluorescence microscopy (Axioplan 2, Zeiss, Germany). And tissue injury was stained by hematoxylin and eosin (H\&E). For immunohistochemistry, sections were then incubated at $4^{\circ} \mathrm{C}$ overnight with anti-mouse MS4a6D(1:100) after the Antigen retrieval. Then apply the corresponding secondary antibody(1:200), and the results were visualized with diaminobenzidine (DAB, Dako). Cellular apoptosis was detected by TUNEL staining (Roche, Berlin, Germany) according to the manufacturer's instructions.

\section{Real-time quantitative RT-PCR}

Total RNA from in vivo-infected livers of each group was extracted using the RNAiso Plus of TaKaRa according to the manufacturer's procedure. The concentration and purity of RNA were detected by using the biological spectrophotometer of Effendorf. Total mRNA $(2 \mathrm{mg})$ was reverse-transcribed into cDNA using the RevertAid H Minus First Strand cDNA Synthesis Kit (Fermentas China, Shenzhen City, China), in accordance with the manufacturer's instructions. The expression of MHV mRNA in tissues was detected using SYBR ${ }^{\circledR}$ Premix Ex Taq $^{\mathrm{TM}}$ II (Tli RNaseH Plus) with cycling parameters as follows: an initial cycle for $2 \mathrm{~min}$ at $95^{\circ} \mathrm{C}$, followed by 40 cycles of $20 \mathrm{~s}$ at $95{ }^{\circ} \mathrm{C}$ and $30 \mathrm{~s}$ at $59{ }^{\circ} \mathrm{C}$. Furthermore, the expression of GAPDH mRNA was assessed simultaneously in all samples, which acted as an internal control. The primer of MHV: Forward-primers

5'-TGGAAGGTCTGCACCTGCTA-3', Reverse-primers 5'-TTTGGCCCACGGGATTG-3'. The primer of GAPDH: Forward-primers 5'GGTGAAGGTCGGTGTGAACG-3',

Reverse-primers

5'-CTCGCTCCTGGAAGATGGTG-3'. The relative mRNA expression was calculated by using the $2^{-\Delta \Delta C T}$ method.

\section{Flow cytometry}

Livers were perfused with PBS through the portal vein to remove blood cell contamination prior to dissection. Liver tissues from the mice were then homogenized, and hepatocytes were removed by sedimentation. Inflammatory cells were enriched using 35\% Percoll gradient (Sigma-Aldrich). Cells were incubated with optimal dilutions of anti-CD45-PE-Cy7, anti-CD11b-PE, isothiocyanate anti-L-D-APC-Cy7,

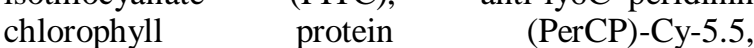


anti-ly6G-allophycocyanin (APC). Stained cells were analyzed on a flow cytometer (FACsAria cytometer, BD, Franklin Lakes, NJ, USA), and the data were processed using FlowJo. Dead cells and doublet cells were excluded on the basis of forward scatter (FSC) and side scatter (SCC), and analyses were performed on 10,000 events recorded. Myeloid cells, gated by high side scatter, were assessed for CD11b and F4/80 to enumerate macrophages $\left(\mathrm{CD}_{11} \mathrm{~b}^{+} \mathrm{F} 4 / 80^{+}\right)$. Ly6C(proinflammatory) and ly6G(anti-inflammatory) expression were assessed to discriminate macrophage.

\section{Statistical analyses}

Statistical analysis of the data was performed using GraphPad Prism 6.0 software. Data were expressed as the mean \pm standard error of the mean. Comparisons between two groups were assessed with Student's t-test, and multiple-group analyses were evaluated by one-way analysis of variance (ANOVA) followed by Fisher's LSD post hoc tests. Survival curves were generated by log-rank test. $\mathrm{P}$-value $\leq 0.05$ were considered significant.

\section{Resules}

MS4a6D is a membrane protein molecule expressed on the surface of antigen presenting cell such as macrophages and DCs.

Most of the expression data obtained by MS4A members to date are mainly limited to organ types, with little information about expression at the cellular level. So far it is known that MS4A1 is expressed on $\mathrm{B}$ cells, and MS4A2 is expressed on mast cells and basophils ${ }^{[16,31,32]}$. MS4A3 and MS4A4 is expressed on hematopoietic cells. Except these genes, the cellular expressions of MS4A6A, MS4A8B, MS4A14 and MS4A15 have also reported ${ }^{[18]}$. However, there are very few studies on MS4a6D at present, and only two related literatures are available. Recently, our experimental group found that MS4a6D gene was expressed in DCs (Dendritic Cells) and Macrophages (Fig.1) by fluorescent dual staining.

In MHV3 infected mice, the deletion of the MS4a6D gene leads to the decrease of macrophages in the liver, in which the pro-inflammatory subsets decreased.

Because MS4a6D are highly expressed on macrophage membranes (Fig.1). Given that macrophages play an important role in fighting acute inflammation. So we next wanted to test the role of the MS4a6D gene in Viral fulminant hepatitis $(\mathrm{FH})$. Groups of 4 C57BL/6 (WT) and MS4a6D knockout $(\mathrm{KO})$ mice of 7 weeks age were injected with 100 PFU of MHV-3 via intraperitoneal injection. At 48 or $72 \mathrm{~h}$ p.i., livers were collected from virus-infected mice of each group. Briefly, liver tissues were isolated and mechanically homogenized, lymphocytes were collected thereafter. Cell suspensions of liver tissues were washed and resuspended in PBS. Then apply the corresponding antibody. Stained cells were analyzed on a FACSAria II flow cytometer, and the data were processed using FlowJo. The outcome reports that the deletion of the MS4a6D gene leads to the decrease of macrophages in the liver, where the pro-inflammatory subgroup $\left(\right.$ ly6C $\mathrm{C}^{+}$) is decreased at $72 \mathrm{~h}$ p.i (Fig 2). While there was little difference at $48 \mathrm{~h}$, which may be because the virus infection time is so short that the differences caused by genetic deletion has not yet been reflected. These results suggest that gene deletion may have the ability to reduce inflammatory damage caused by $\mathrm{FH}$ infection.

\section{The symptoms of tissue necrosis were improved in} MHV3 infected MS4a6D ${ }^{-/-}$mice.

To test this hypothesis, we also used haematoxylin-eosin (HE) staining to detect pathological changes in liver tissues. After the mice were infected with MHV3 virus for $48 \mathrm{~h}$ and $72 \mathrm{~h}$, the liver tissue was taken out and fixed in $4 \%$ paraformaldehyde for $30 \mathrm{~h}$. The samples were then dehydrated in increasing concentrations of ethanol-xylene and embedded in paraffin. The paraffin blocks obtained were cut into $3 \mu \mathrm{m}$ slices, and placed on the glass, and then the samples were deparaffinized and rehydrated. The sections were stained with haematoxylin and eosin (HE) according to the standard procedures. Stained sections were examined under the microscope at 200x magnification and photographed. HE results showed that $\mathrm{MS} 4 \mathrm{a} 6 \mathrm{D}^{-1-}$ mice manifested with less severe fibrinogen deposition, liver injury and hepatocyte necrosis (Fig.3). This suggests that the deletion of MS4a6D gene has a protective effect on $\mathrm{FH}$ mice. The MS4a6D gene aggravates inflammatory infiltration and tissue necrosis in FH mice.

\section{There was no statistical difference in mortality between WT and MS4a6D KO mice infected with MHV3.}

Since the pro-inflammation macrophages and the replication of virus was decreased after the MS4a6D gene was knockout, we wanted to test whether the survival rates of the MS4a6 $\mathrm{D}^{-/-}$mice would change compare with the WT after exposure to MHV3. Two groups of mice were prepared, one with MS4a6D gene knockout and the other with wild type. Two groups of mice were infected with $100 \mathrm{PFU}$ of MHV-3 via intraperitoneal injection. Then we recorded the survival of the mice and performed a statistical analysis. As shown in the fig.4, the survival rate of $\mathrm{MS} 4 \mathrm{a} 6 \mathrm{D}^{-/}$is higher than WT mice, while there were no statistical differences.

\section{Discussion}

In vitro transfection studies suggests that MS4A proteins may play an important role in cellular signaling, including ion channel activity that is associated with cell cycling and differentiation ${ }^{[19-22]}$. Although it is increasingly evident that the MS4A family has important and diverse roles throughout the 
body, the precise functions of many MS4A proteins remain enigmatic, especially MS4a6D.

At present, there are very few researches on MS4a6D, and only two related articles have reported the gene. At the cellular level, we detected the expression of MS4a6D gene on macrophages and DCs by immunofluorescence(Fig.2). During acute infection, it has been suggested that the balance between protection and liver damage regulated by the host's immune response will be the decisive factor in the outcome of hepatitis. Since MS4a6D is expressed on macrophages. Acute hepatitis always threatens people's health and life. And it is well known that macrophages play an important role in fighting acute viral hepatitis. So we wanted to further verify the role of the MS4a6D gene in hepatitis, in order to discover the function of the gene and lay the foundation for finding therapeutic drugs for hepatitis.

In MHV3 infected mice, we found that the deletion of the MS4a6D gene leads to an decrease of macrophages in the liver, in which the pro-inflammatory macrophage subsets decreased. In addition, the replication of viral is lower than WT. Histopathological analysis of the liver revealed less and smaller necrotic foci in MHV3-infected MS4a6D ${ }^{-1-}$ mice than WT mice. Although the absence of the gene attenuated symptoms of inflammation, there was no difference in survival rates compared with WT mice. It is probably because MHV3 is so toxic that the differences between the two groups have been masked. In the model of a chronic virus, the survival rate may be more significantly affected by the reduced inflammation. This hypothesis requires further research. And the mechanism of MS4a6D affecting the number and function of macrophages is not well understood. Moreover, since MS4a6D is expressed on DC cells, what is its effect on DCs? These puzzles need to do more work.

In summary, the results obtained indicate that MS4a6D may aggravate the immunopathological damage when infected with hepatitis virus. It suggest that inhibition of MS4a6D signaling pathway by immunotherapy, in combination with blocking of other inflammatory factors may be an effective treatment for $\mathrm{FH}$ and other severe inflammatory diseases.

\section{Conflict of Interest}

The authors declare no conflict of interest.

\section{References}

1. Sarin, S.K., et al., Acute-on-chronic liver failure: consensus recommendations of the Asian Pacific Association for the study of the liver (APASL). Hepatology International, 2009. 3(1): p. 269.

2. Rosen, H.R. and P. Martin, Viral hepatitis in the liver transplant recipient. Infect Dis Clin North Am, 2000. 14(3): p 761-784.
3. Chen, Y., et al., Programmed death (PD)-1-deficient mice are extremely sensitive to murine hepatitis virus strain-3 (MHV-3) infection. Plos Pathogens, 2011. 7(7): p. e1001347.

4. Pope, M., et al., Pattern of disease after murine hepatitis virus strain 3 infection correlates with macrophage activation and not viral replication. Journal of Virology, 1995. 69(9): p. 5252-60.

5. Levy, G.A., J.L. Leibowitz, and T.S. Edgington, Induction of monocyte procoagulant activity by murine hepatitis virus type 3 parallels disease susceptibility in mice. Journal of Experimental Medicine, 1981. 154(4): p. 1150-1163.

6. Lucchiari, M.A., et al., Acquired immunity of $\mathrm{A} / \mathrm{J}$ mice to mouse hepatitis virus 3 infection: dependence on interferon-gamma synthesis and macrophage sensitivity to interferon-gamma. Journal of General Virology, 1991. 72 ( $\mathrm{Pt}$ 6)(72 ( Pt 6)): p. 1317

7. Guo, S., et al., The NLRP3 Inflammasome and IL-1 Accelerate Immunologically Mediated Pathology in Experimental Viral Fulminant Hepatitis. Plos Pathogens, 2015. 11(9): p. e1005155.

8. Kinet, J.P., et al., Isolation and characterization of cDNAs coding for the beta subunit of the high-affinity receptor for immunoglobulin E. Proceedings of the National Academy of Sciences of the United States of America, 1988. 85(17): p. 6483-6487.

9. Tedder, T.F., et al., Isolation and Structure of a cDNA Encoding the B1 (CD20) Cell-Surface Antigen of Human B Lymphocytes. Proc Natl Acad Sci U S A, 1988. 85(1): p. 208-212.

10. Adra, C.N., et al., Cloning of the cDNA for a Hematopoietic Cell-Specific Protein Related to CD20 and the $\beta$ Subunit of the High-Affinity IgE Receptor: Evidence for a Family of Proteins with Four Membrane-Spanning Regions. Proc Nat Acad Sci U S A, 1994. 91(21): p. 10178-10182.

11. Hulett, M.D., E. Pagler, and J.R. Hornby, Cloning and characterization of a mouse homologue of the human haematopoietic cell-specific four-transmembrane gene HTm4 Immunology \& Cell Biology, 2001. 79(4): p. 345-9.

12. Ishibashi, K., et al., Identification of a new multigene four-transmembrane family (MS4A) related to CD20, HTm4 and $\beta$ subunit of the high-affinity IgE receptor. Gene, 2001. 264(1): p. 87-93

13. Liang, Y. and T.F. Tedder, Identification of a CD20-, FcepsilonRIbeta-, and HTm4-related gene family: sixteen new MS4A family members expressed in human and mouse. Genomics, 2001. 72(2): p. 119-127.

14. Polyak, M.J., S.H. Tailor, and J.P. Deans, Identification of a cytoplasmic region of $\mathrm{CD} 20$ required for its redistribution to a detergent-insoluble membrane compartment. Journal of Immunology, 1998. 161(7): p. 3242-3248.

15. Tedder, T.F., et al., Structure of the gene encoding the human B lymphocyte differentiation antigen CD20 (B1). Journal of Immunology, 1989. 142(7): p. 2560.

16. Beers, S.A., et al., CD20 as a Target for Therapeutic Type I and II Monoclonal Antibodies. Seminars in Hematology, 2010. 47(2): p. 107-114.

17. Zloh, M., D. Esposito, and W.A. Gibbons, Spectroscopy-Based Modelling of the 3D Structure of the $\beta$ Subunit of the High Affinity IgE Receptor. Molecular Simulation, 2000. 24(4-6): p. 421-447.

18. Li, E.K., et al., The MS4A family: counting past 1, 2 and 3 . Immunology \& Cell Biology, 2016. 94(1).

19. Koslowski, M., et al., MS4A12 is a colon-selective store-operated calcium channel promoting malignant cell processes. Cancer Research, 2008. 68(9): p. 3458-3466.

20. Bubien, J.K., et al., Transfection of the CD20 cell surface molecule into ectopic cell types generates a $\mathrm{Ca} 2+$ conductance found constitutively in B lymphocytes. Journal of Cell Biology, 1993. 121(5): p. 1121-1132.

21. Xu, H., et al., MS4a4B, a CD20 homologue in $\mathrm{T}$ cells, inhibits $\mathrm{T}$ cell propagation by modulation of cell cycle. Plos One, 2010. 5(11): p. e13780.

22. Michel, J., et al., Identification of the novel differentiation marker MS4A8B and its murine homolog MS4A8A in 
colonic epithelial cells lost during neoplastic transformation in human colon. Cell Death \& Disease, 2013. 4(1): p. e469.

23. Greer, P., et al., A Family of non-GPCR Chemosensors Defines an Alternative Logic for Mammalian Olfaction. Cell, 2016. 165(7): p. 1734-1748.

24. Hasan, M., et al., Novel genes in brain tissues of EAE-induced normal and obese mice: Upregulation of metal ion-binding protein genes in obese-EAE mice. Neuroscience, 2016. 343: p. 322.

25. Klevens, R.M., et al., Estimating acute viral hepatitis infections from nationally reported cases. American Journal of Public Health, 2014. 104(3): p. 482-7.

26. Lavanchy, D., The global burden of hepatitis C. Liver International, 2010. 29(s1): p. 74-81.

27. Klevens, R.M., et al., The Evolving Epidemiology of Hepatitis A in the United States: Incidence and Molecular Epidemiology From Population-Based Surveillance,
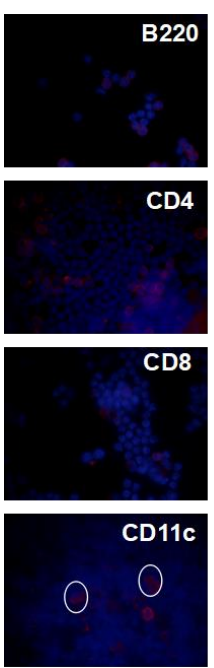
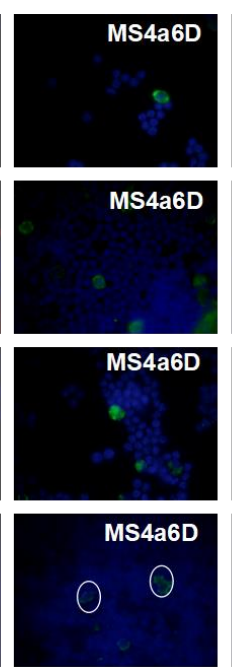
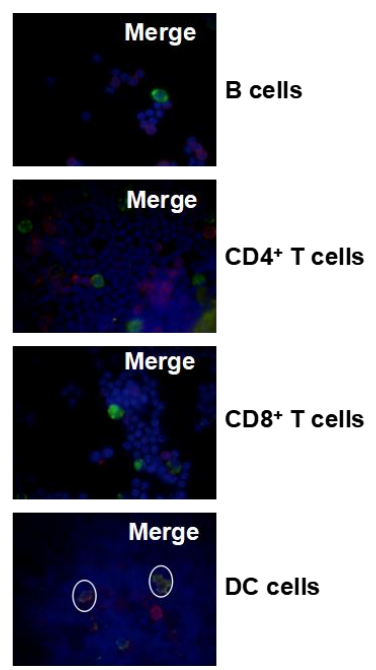

2005-2007. Archives of Internal Medicine, 2010. 170(20): p 1811.

28. Zuccolo, J., et al., Phylogenetic Analysis of the MS4A and TMEM176 Gene Families. Plos One, 2010. 5(2): p. e9369.

29. Brown, K., J. Turton, and K. Morgan, Membrane-Spanning 4-Domains Subfamily A, MS4A Cluster. 2013.

30. Bangur, C.S., et al., Identification and characterization of L985P, a CD20 related family member over-expressed in small cell lung carcinoma. International Journal of Oncology, 2004. 25(6): p. 1583-1590.

31. Glennie, M.J., et al., Mechanisms of killing by anti-CD20 monoclonal antibodies. Molecular Immunology, 2007. 44(16): p. 3823-3837.

32. Lin, S., et al., The FceRI $\beta$ Subunit Functions as an Amplifier of FceRI $\gamma$-Mediated Cell Activation Signals. Cell, 1996. 85(7): p. 985-995.

Figure 1. The expression of MS4a6D on Macrophages and DCs.

Confocal microscopic analysis of the expression of MS4a6D on several cell types. All cell subtypes and MS4a6D were stained by marker with red and green fluorescence. If the two fluorescence combinations turn yellow, it's proved that MS4a6D is expressed on this cell subgroup. Scale bar $=20 \mathrm{~mm}$. Magnification $\times 200$. 
A
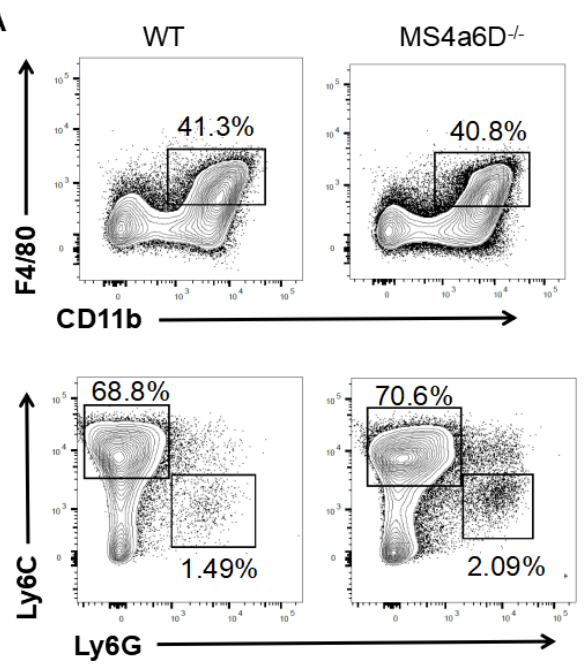

B
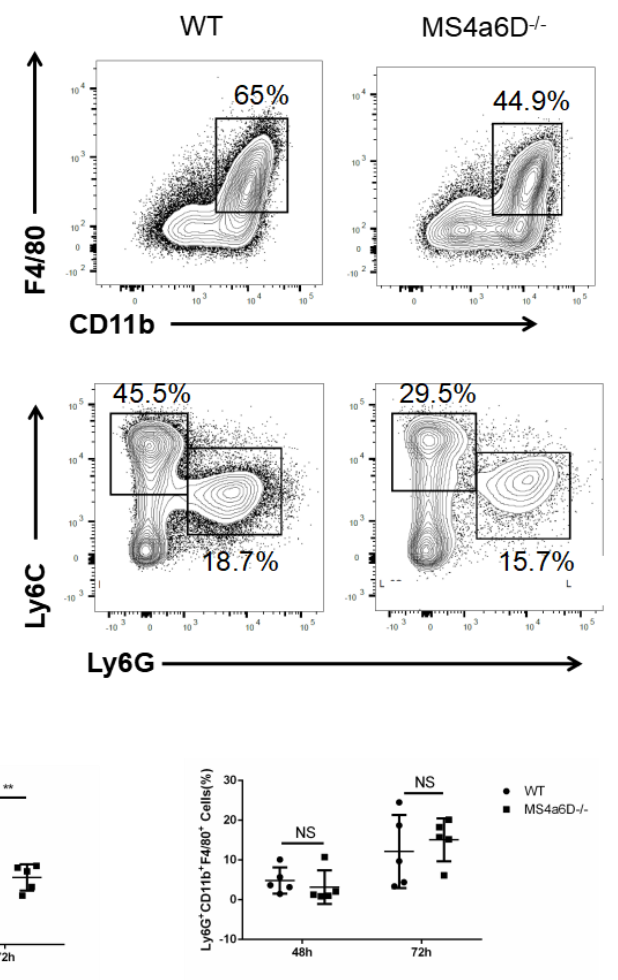

Figure 2. Development of macrophages at different time points in the liver.

(A) Shown are contour plots (top panel) of $\mathrm{CD} 11 \mathrm{~b}^{+} \mathrm{F} 4 / 80^{+}$macrophages among $\mathrm{CD} 45^{+}$cells at 48 hours post infection. Flow-cytometric analyses of Ly6C and Ly6G-binding (bottom panel) macrophages at 48 hours.

(B) Analysis (top panel) of $\mathrm{CD} 11 \mathrm{~b}^{+} \mathrm{F} 4 / 80^{+}$macrophages among CD $45^{+}$cells at 72 hours post infection. And shown

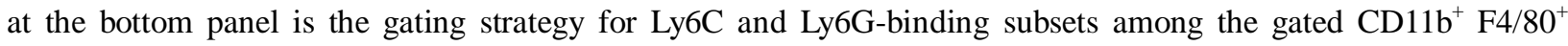
macrophages.

(C) Statistics of total macrophages and its subsets $\left(\mathrm{Ly}_{6} \mathrm{C}^{+}\right.$and $\left.\mathrm{Ly} 6 \mathrm{G}^{+}\right)$at 48 and $72 \mathrm{~h}$ after MHV3 infection, respectively. Data are means \pm SEM (E) and are representative of two independent experiments with five mice per group. ${ }^{*} \mathrm{p}<0.05 ; * * \mathrm{p}<0.001 ; * * * \mathrm{p}<0.0001 ;$ NS: no significant difference. (unpaired Student's $\mathrm{t}$ test). 
A

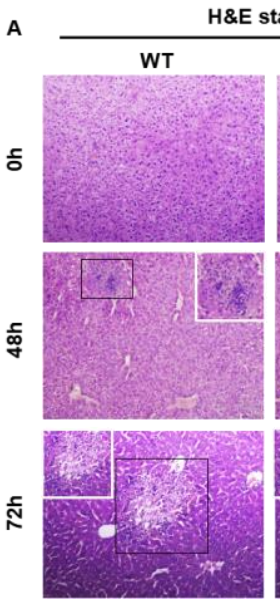

H\&E staining
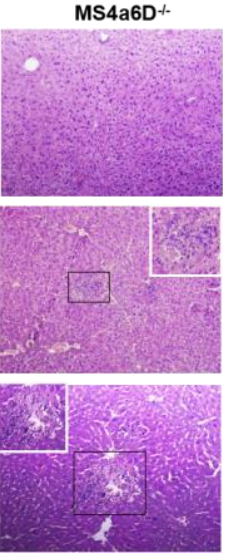

TUNEL
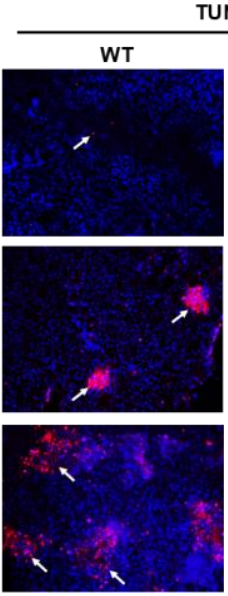
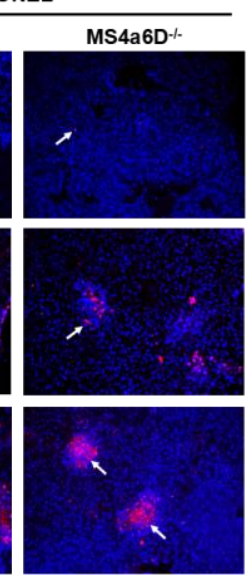

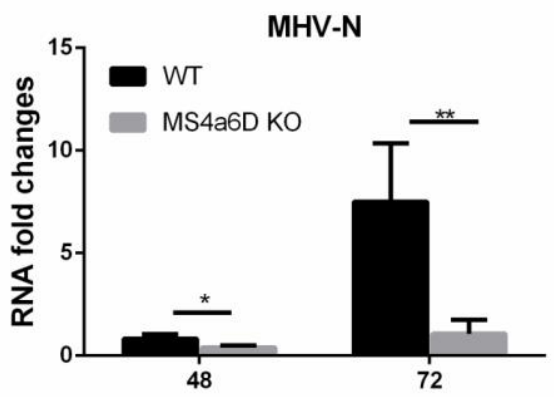

Hours post infection (hrs)

Figure 3. Hepatic damage and viral replication in MHV3-infected WT and MS4a65 ${ }^{-/}$mice.

(A)H\&E staining of liver, and TUNEL staining of cell apoptosis in the liver ( $0 \mathrm{~h}, 48 \mathrm{~h}$ and $72 \mathrm{~h})$ from WT and MS4a6 $^{-/-}$mice after MHV-3 infection, scale bar $=20 \mu \mathrm{m}, \mathrm{n}=5-8$ per group, arrow indicated positive cells.(B) MHV3 replication in livers from each group of infected mice was determined by analysis of the nucleoprotein (NP) RNA expression at 48 and $72 \mathrm{~h}$ p.i. by qRT-PCR

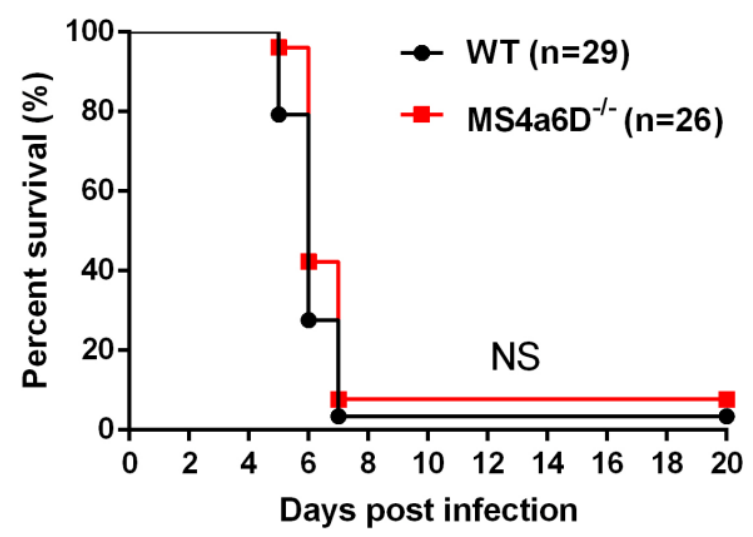

Figure 4. The deletion of MS4a6D had no effect on mortality of FH MICE.

The survival rate of virus infected mice was monitored for a total of 20 days. One representative of four experiments with similar results is shown. $\mathrm{p}<0.05$ was considered significant different; NS: no significant difference. 